\title{
BMJ Open Quality Reducing unnecessary delays during the transfer of patients from the paediatric intensive care unit to the general ward: a quality improvement project
}

\author{
Hamza Alali, ${ }^{1,2}$ Yasser Kazzaz, ${ }^{\oplus 1,2,3}$ Ali Alshehri, ${ }^{1,2,3}$ Mohannad Antar, ${ }^{1}$ \\ Ousaima Alhamouieh, ${ }^{4}$ Zahra Hasan, ${ }^{5}$ Khaled Al-Surimi ${ }^{6,7}$
}

To cite: Alali H, Kazzaz Y, Alshehri A, et al. Reducing unnecessary delays during the transfer of patients from the paediatric intensive care unit to the general ward: a quality improvement project. BMJ Open Quality 2019;8:e00695. doi:10.1136/ bmjoq-2019-000695

Received 26 March 2019 Revised 31 July 2019 Accepted 13 August 2019
Check for updates

(C) Author(s) (or their employer(s)) 2019. Re-use permitted under CC BY-NC. No commercial re-use. See rights and permissions. Published by BMJ.

For numbered affiliations see end of article.

Correspondence to

Dr Hamza Alali;

drhamza80@hotmail.com

\section{ABSTRACT}

Introduction Delaying the discharge of paediatric intensive care unit (PICU) patients is directly proportional to increased occupancy rate and cost. We aimed to study the process of transferring patients from the PICU to the general ward in order to improve the timeliness of this process while guaranteeing patient safety.

Methods A multidisciplinary quality improvement (QI) team was formed to analyse the transfer process. Several Plan Do Study Act cycles were tested, targeting all steps of the transfer process, and applying turnaround time (TAT) the duration from the time of clinical transfer decision until the physical transfer of the patient-as an outcome measure, aiming for a TAT of 4 hours.

Results Baseline results showed that medical transfer decisions by PICU attending physicians were taken late for most patients: only $19 \%$ of decisions were made by 08:00 by the on-call team. Average TAT of the transfer process was over 7 hours, with duration ranging from 7 to 17 hours. After implementing all suggested improvement interventions, early decision compliance improved to $59 \%$. TAT improved gradually, starting in January 2017 , until it approached our target (284-261 $\mathrm{min} \approx 4$ hours) in February-May 2017.

Conclusion PICU patient transfer process delays can be reduced by early evaluation, timely team communication and proper preparation. It is recommended that all personnel with early involvement avoid unnecessary delays by paying more attention to all process steps, starting with the clinical decision, until the physical transfer. Standardising transfer processes might lead to a decrease in the length of PICU stay, which is a desirable outcome, but this observation needs further exploration.

\section{BACKGROUND AND QUALITY PROBLEMS}

Critical care service is an essential component of the modern healthcare system; it provides immediate high-acuity interventions for life-threatening conditions, and close monitoring for postoperative high-risk patients. Unfortunately, intensive care unit (ICU) beds are restricted in many hospitals, due to high cost and limited resources and experienced staff. Therefore, a rationalised utilisation of resources and time is needed. ${ }^{12}$ Unnecessary
ICU patient stays are a major contribution to poor bed utilisation; which is affected by many factors, from clinical decisions to non-clinical processes such as communication systems, and bed availability in the general ward. ${ }^{34}$ Evidence from previous studies showed that unnecessary delays in transferring patients were associated with increased ICU occupancy rate and cost. ${ }^{56}$ After struggling with the number of paediatric intensive care unit (PICU) patients admitted to the emergency room under critical care services with no beds available in our PICU, there was a vital need for transfer process evaluation and optimisation. A multidisciplinary quality improvement (QI) team was formed, and a checklist was created to monitor transfer process timelines. Baseline data results showed an average transfer process turnaround time (TAT) of more than 7 hours, with duration ranging from 7 to 17 hours. The international benchmark for transfer duration (from clinical decision to physical transfer) is 4 hours. ${ }^{7}$

\section{PROJECT CONTEXT}

This QI project was conducted at King Abdullah Specialized Children's Hospital (KASCH), an academic governmental tertiary hospital in King Abdulaziz Medical City, Riyadh, Saudi Arabia. The hospital consists of 214 paediatric inpatient beds and 20 medical-surgical PICU beds. The paediatric critical care unit is run by attending, fellows, specialists and residents. The nurse to patient ratio is 1-2:1. The average admission number during the project period (August 2016-May 2017) was 80 patients per month, with an average occupancy rate of $81 \%$. We aimed to study the processes and duration of patient transfers from the PICU to the general ward in KASCH to identify possible gaps causing improper transfer, and to improve the timeliness of the process while guaranteeing patient safety. 


\section{IMPROVEMENT METHODS}

Lean and Six Sigma was the methodology adopted to run this QI project. ${ }^{89}$ In June 2016, a multidisciplinary QI team was formed by two PICU consultants, an assistant consultant, a nurse coordinator and a QI specialist, in order to work collaboratively to standardise transfer process. The details of the adopted methodology in the improvement journey were addressed under the acronym of the Six Sigma approach Define, Measure, Analyse, Improve and Control.

\section{Define step}

A process map of the patient transfer journey was established, with a time frame for each step. Those steps included:

1. Transfer decision time.

2. Ward bed allocation.

3. Communication with the receiving team physician in charge of PICU transfers.

4. Bedside evaluation and writing of transfer orders by the receiving team physician.

5. Nurses' communication and patient preparation until the physical transfer.

Data were continuously collected and analysed; reasons for delays were identified; and several new ideas were implemented at each step in order to reach the target.

\section{Measure step}

In order to improve our process, it was important to define baseline measurements. A transfer checklist was developed to measure the total duration of the transfer process, as well as the duration for each process step. The checklist was filled out by the primary physician (resident or fellow), and rechecked and completed by the primary nurse, on the patient's physical transfer. The outcome measure was the total transfer time from the clinical transfer decision by the PICU attending physician, until the patient was physically transferred to the general ward. The goal statement was, "to ensure safe and efficient patient transfer processes from the PICU to the general ward within fourhours by May 2017". Process measures included the duration of each step in the transfer process, which was recorded in the checklist. A delay in any step was targeted by an intervention to optimise its time, and thus the overall time (figure 1).

\section{Analyse step}

To identify the root causes of delayed transfer, we analysed all transfer steps and calculated the time intervals for each step to find bottlenecks that were leading to suboptimal performance. Data were analysed on a monthly basis by the assigned QI team.

\section{Improve step}

The identified problems were late transfer decisions during the day, delays in bed allocation, delays in contacting the receiving team, delays in writing transfer orders by the receiving team, and delays in writing transfer notes by the PICU team. Several Plan Do Study Act cycles

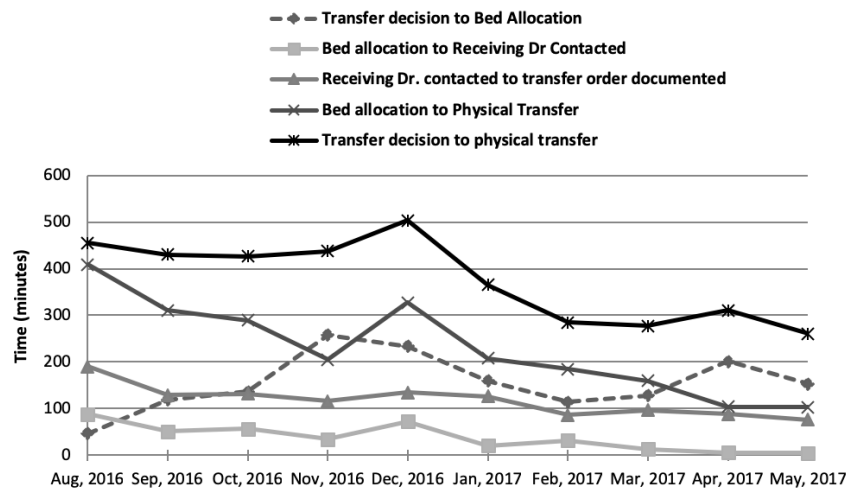

Figure 1 Average time interval in minutes for each step of the transfer process per month with overall transfer time (transfer decision to physical transfer).

(PDSAs) were performed to improve the current practice; these interventions included early evaluation for potentially transferable patients by the PICU on-call team, early bed allocation if possible, timely communication with the primary physician to document transfer orders in patient electronic medical records (EMR), and encouraging nurses to start preparations for patient disposition as early as possible. Further details about these PDSAs are explained in the improvement strategy section (figure 2).

\section{Control step}

Following implementation of all changes, the project achieved its intended outcome. In order to sustain this achievement, the PICU team decided to define transfer process steps (writing transfer summaries, informing bed management departments about bed allocation, communicating with receiving physicians and preparing patient staff) in parallel model. Departmental policies and procedures for PICU patient transfers were established to reinforce the new practices and ensure strict compliance by all involved parties. Another control measure was the implementation of the final version of the transfer checklist as a mandatory departmental form in patients' EMR. Transfer duration time was suggested as one of the unit's key performance indicators, but remained under review.

\section{IMPROVEMENT STRATEGY}

There were five PDSA cycles.

\section{PDSA 1 of early transfer decision}

Before the project began, patients who were potential transfers were assessed late (during or after the daily rounds from 09:00 to 12:00). PICU team leaders agreed that all potential transfers should be evaluated in the early morning by the on-call team, with decisions made before 08:00. A list of transferable patients would then be sent to the bed management office responsible for bed allocations in the hospital. Transfer decision time was monitored, and data showed an improvement in physician compliance with earlier decision-making (from 19\% in June through July 2016, to 59\% in May 2017). 


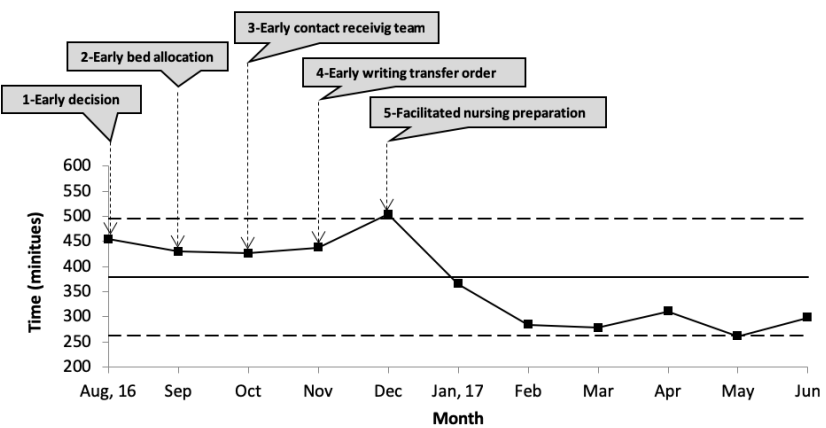

Figure 2 Impact of improvement interventions on the overall time of the transfer process.

\section{PDSA 2 of early bed allocation}

Bed allocation for PICU patients in the general ward is controlled by the bed management department, following hospital policies regarding bed distribution and utilisation.

To emphasise the need for early bed allocation, a meeting with the bed management team was arranged regarding when and how to prioritise the transfer of PICU patients. The time from transfer decision to bed allocation was monitored during the project. The PICU charge nurse sent the patient transfer list earlier in the morning (before 08:00) to the bed management office in order to facilitate earlier bed allocation (figure 1).

\section{PDSA 3 of communication with the receiving team}

Another step-in reducing time delays was earlier communication with receiving physicians, between 08:30 and 09:00 (the time between handover and the beginning of clinical rounds in paediatric wards). The time until the receiving physicians were contacted decreased gradually over the project period (figure 1).

\section{PDSA 4 of physical assessment and documenting transfer} orders in patient EMR by the receiving physician

To encourage participation from other departments regarding the documentation of early transfer assessments and orders, training was conducted. Receiving teams were requested to enter transfer orders in EMRs within 1 hour of notification by the PICU team. As the project proceeded, receiving teams took less time to attend to, evaluate, and document transfer orders (figure 1).

\section{PDSA 5 of patient preparation until physical transfer}

This cycle targeted the last step of the process: the physical transfer of the patient to the general ward after documentation of the transfer order in the EMR and bed allocation in the ward. This intervention was under the scope of the nursing team. It was very important for the QI team to empower nurses, and to educate the nurse manager and the charge nurses, about their proactive roles (timely communication with the primary receiving nurse to ensure readiness of ward room, preparation of medications, and readiness of social workers and family members) in order to prevent the occurrence of any delay. This intervention was monitored by the time interval of bed allocation to patient physical transfer, which showed a gradual decrease as the project proceeded (figure 1).

\section{RESULTS}

Transfer decisions were made earlier (before 08:00) by $59 \%$ (40/68 transfers on May 2017) after implementation of improvement interventions. Before the project started, this figure was only 19\% (9/47 transfers in June and July 2016). The average time interval of each step improved gradually. The time between the transfer decision and bed allocation decreased from $257 \mathrm{~min}$ (its highest value, in November 2016) to $153 \mathrm{~min}$ (at the end of the project). Time intervals of bed allocation until receiving doctor is contacted, receiving doctor documented transfer order in the EMR and timing from bed allocation to physical transfer decreased from 88 to $5 \mathrm{~min}, 190$ to $76 \mathrm{~min}$ and 408 to $103 \mathrm{~min}$, consecutively, by the end of the project in May 2017, compared with timings at the beginning of the project in August 2016 (figure 1). At the project outcome level, average TAT started to improve noticeably in January 2017, and approached our target of 261 min $\approx 4$ hours in May 2017 (figure 2).

\section{DISCUSSION}

Timely transfer of patients from the ICU to the general ward is one of the specific aspects of handover which showed a positive change following intervention. Although optimal patient flow is critical to ensure high quality patient care, there is no clear evidence about the best way to achieve an ideal, safe and efficient patient transfer out of the ICU, especially in the paediatric population. ${ }^{1011}$

One report, from C.S. Mott Children's Hospital at the University of Michigan, evaluated and analysed the PICU patient transfer process and concluded that communication-related bottlenecks include conflicting variables. Analyses showed delays in paging the receiving unit by Admissions and Bed Coordination Centre (165 min), and in the response from the receiving unit regarding bed allocation $(74 \mathrm{~min})$. This report did not evaluate the total process that begins with the clinical transfer decision and ends with the physical transfer of the patient. ${ }^{12}$

Although our institution had PICU admission and discharge guidelines in place, they were not clear and were not followed most of the time, resulting in ineffective and delayed patient transfers, as showed in baseline data. In this QI project, we elected to focus on decreasing time delays to release the pressure and demand on PICU beds from other services, such as emergency departments, operating rooms and general wards.

At the beginning, we had to meet teams involved in the transfer process, such as PICU physicians and nurses, to emphasise the early transfer decision and the importance of sending the transfer list to bed management office, paediatric residency programme and 
department/division heads for a prompt response in evaluating patients, and entering transfer orders in the EMR within 1 hour. An unsteady improvement in each step was achieved, but during the first 2 months of winter, total transfer time increased due to increased hospital occupancy rate and the difficulty in allocating beds to transferring PICU patients. More effort was needed on an administrative level to facilitate evacuation of beds in the ward to better accommodate PICU transfers. After all interventions were in place, a modified transfer process was needed to implement the changes. A parallel transfer process, with summaries written 1 day ahead, was recommended. Some steps such as bed allocations were difficult to control, as bed management department oversees the flow of all patients and distribute beds based on existing policies, which may prioritise emergency patients over ICU transfers. Another difficulty was the assessment of the patient by the receiving team during morning rounds. The solution to this bottleneck was allocating a board-certified physician to be responsible for PICU transfer decisions, and for entering transfer orders into the EMR. Time intervals were analysed after each intervention to test its effect on the whole process. The overall time of the process did not show an improvement until all interventions took place. Family impacts on the process were not explored in depth through the project, as PICU parents are not allowed to stay in the unit. In some cases (like those transferred from the PICU to a high dependency unit), a child can be moved without his or her family present, but the family should be informed. Only a few projects described the ICU transfer process from the nursing perspective ${ }^{11} 13$ in adult ICU patients, but this QI project described the entire process, from medical decision to physical transfer, and provided specific analyses for paediatric ICU transfer steps; proposing a new transfer process to avoid PICU transfer delays and optimise patient flow.

\section{Lessons learned}

This project led to indirect positive impacts systemwide, reflected in PICU length of stay (LOS) and cost. ICU LOS is a key measure for resource utilisation, and it affects the patient, the family and the entire healthcare system. ${ }^{14} 15$ Although many factors affect PICU LOS, we

\section{PICU LOS control chart}

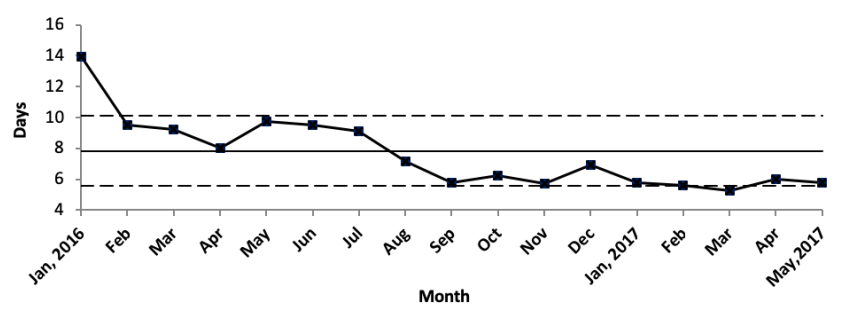

Figure 3 PICU average length of stay per month before and during the project period. LOS, length of stay; PICU, paediatric intensive care unit. noticed a decrease in our PICU LOS during the project period (figure 3). This observation may be related to increased awareness of timely transfers, and the active involvement of the entire team in discharging patientsbut this impact needs more exploration.

This project has shown good business sense. The cost was calculated as PICU day/patient versus ward day/ patient in US dollar, based on our local institution's financial regulations. A time period longer than 4 hours in PICU after the transfer decision was made was considered a delay in transfer. This calculation considered the delayed hours for transferred patients on the day of physical transfer only; days delayed due to bed unavailability were not considered. Wasted costs decreased almost tenfold (US $\$ 6431$ on December 2016, to US $\$ 622$ on May 2017).

We learnt that bundling improvement intervention is effective, and overall aims cannot be achieved until all steps involved in the process are improved (in other words, all or none). The involvement of concerned stakeholders, leaders and decision makers played a great role in executing the interventions needed. It is necessary that each improvement have official management support through approved policies, memos, or guidelines to direct the process and keep it on track.

\section{Challenges and limitations}

This project had some challenges due to the necessary cooperation of many different services, including the general paediatric department, the surgery department, the residency programme, bed management, nursing services, housekeeping services and social services. The improvement phase started with a seasonal winter crisis and a high rate of PICU occupancy, which added another challenge to obtaining clinicians' commitment to the process, especially bed allocation in the general ward. Weekends, holidays and after-hours times were also challenging, due to staff already working on call. Families' desires to accompany their children during transfer added an extra delay in some cases. Although this QI project has been done in PICU of a big and referenced hospital in Saudi Arabia, its findings could not be generalisable across other PICUs due to system differences.

\section{CONCLUSION}

Time delays in paediatric ICU patient transfers can be reduced by early evaluation, timely team communication and proper preparation. It is recommended that all personnel with early involvement avoid unnecessary delays by paying more attention to all process steps, starting with the clinical decision, until the physical transfer. Standardising the transfer process may lead to a decrease in PICU LOS, which is a desirable outcome. However, this observation needs further exploration.

Author affiliations

${ }^{1}$ Department of Pediatrics, Ministry of the National Gaurd-Health Affairs, Riyadh, Saudi Arabia 
${ }^{2}$ King Abdullah International Medical Research Center, Riyadh, Saudi Arabia

${ }^{3}$ College of Medicine, King Saud bin Abdulaziz University for Health Sciences, Riyadh, Saudi Arabia

${ }^{4}$ Quality and Patient Safety Department, Ministry of the National Gaurd-Health Affairs, Riyadh, Saudi Arabia

${ }^{5}$ Nursing Services, Ministry of the National Gaurd-Health Affairs, Riyadh, Saudi Arabia

${ }^{6}$ Public Health and Health Informatics, King Saud bin Abdulaziz University for Health Sciences, Riyadh, Saudi Arabia

${ }^{7}$ Primary Care and Public Health, Imperial college London, London, United Kingdom

Acknowledgements We would like to appreciate all departments supported this achievement, including paediatric intensive care, paediatric residency programme in King Saud bin Abdulaziz University for health sciences, Bed Management department and Nursing services in King Abdullah Specialized Children Hospital of King Abdulaziz Medical City, Riyadh. We would like to thank Editage (www.editage. com) for English language editing.

Contributors HA: Quality improvement project team leader, project conduction, manuscript writing.YK: Quality improvement project team co-leader, data analysis, interventions planning and conducting, manuscript review. AA: PICU head division, improvement interventions conduction, manuscript review. MA: Quality improvement project team member, follow project progress, data collection, introduction and discussion writing. OA, Ql specialist: Quality improvement project team member, data analysis, methodology writing. ZH: Quality improvement project team member, nursing related interventions conduction, data collection. KA-S: Project mentor, methodology writing, manuscript edit. HA (team leader) is responsible for the overall content in this manuscript.

Funding The authors have not declared a specific grant for this research from any funding agency in the public, commercial or not-for-profit sectors.

Competing interests None declared.

Patient consent for publication Not required.

Ethics approval This project was approved by the hospital's quality improvement and patient safety department and the Instititutional Review Board (IRB).

Provenance and peer review Not commissioned; externally peer reviewed.

Open access This is an open access article distributed in accordance with the Creative Commons Attribution Non Commercial (CC BY-NC 4.0) license, which permits others to distribute, remix, adapt, build upon this work non-commercially, and license their derivative works on different terms, provided the original work is properly cited, appropriate credit is given, any changes made indicated, and the use is non-commercial. See: http://creativecommons.org/licenses/by-nc/4.0/.

\section{REFERENCES}

1. Ward NS, Chong DH, Beds CC. And resource utilization: current trends and controversies. Semin Respir Crit Care Med 2015;36:914-20.

2. Turner EL, Nielsen KR, Jamal SM, et al. A review of pediatric critical care in resource-limited settings: a look at past, present, and future directions. Front. Pediatr. 2016;4.

3. Lin F, Chaboyer W, Wallis M. A literature review of organisational, individual and teamwork factors contributing to the ICU discharge process. Aust Crit Care 2009;22:29-43.

4. Fieldston ES, Li J, Terwiesch C, et al. Direct observation of bed utilization in the pediatric intensive care unit. $J$ Hosp Med 2012;7:318-24.

5. Chrusch CA, Olafson KP, McMillan PM, et al. High occupancy increases the risk of early death or readmission after transfer from intensive care. Crit Care Med 2009;37:2753-8.

6. Johnson DW, Schmidt UH, Bittner EA, et al. Delay of transfer from the intensive care unit: a prospective observational study of incidence, causes, and financial impact. Critical Care 2013;17.

7. Secretariat CC. Critical Care Unit Balanced Scorecard Toolkit. Secondary Critical Care Unit Balanced Scorecard Toolkit, 2012. Available: https://www.criticalcareontario.ca/FR/Toolkits/Tableau\% 20de\%20bord\%20\%C3\%A9quilibr\%C3\%A9\%20(2012).pdf

8. Schweikhart SA, Dembe AE. The applicability of lean and six sigma techniques to clinical and translational research. J Investig Med 2009;57:748-55.

9. Silich SJ, Wetz RV, Riebling N, et al. Using six sigma methodology to reduce patient transfer times from floor to critical-care beds. $J$ Healthc Qual 2012;34:44-54.

10. van Sluisveld N, Hesselink G, van der Hoeven JG, et al. Improving clinical handover between intensive care unit and general ward professionals at intensive care unit discharge. Intensive Care Med 2015;41:589-604.

11. Chaboyer W, Thalib L, Foster M, et al. The impact of an ICU liaison nurse on discharge delay in patients after prolonged ICU stay. Anaesth Intensive Care 2006;34:55-60.

12. University of Michigan health system Paoa. Analysis of PICU patient transfer process: University of Michigan, 2014. Available: http:// umich.edu/ ioe481/ioe481 past reports/F1402.pdf

13. Chaboyer W, Lin F, Foster M, et al. Redesigning the ICU nursing discharge process: a quality improvement study. Worldviews Evid Based Nurs 2012;9:40-8.

14. Gruenberg DA, Shelton W, Rose SL, et al. Factors influencing length of stay in the intensive care unit. Am J Crit Care 2006;15:502-9.

15. Pollack MM, Holubkov R, Reeder R, et al. PICU length of stay: factors associated with bed utilization and development of a benchmarking model. Pediatr Crit Care Med 2018;19:196-203. 